\title{
Generation of micro channels in AlMg4.5Mn0.7 sheets using a vibration-assisted micro forming process
}

\author{
Benedikt Müller* and Andreas Schubert \\ Chemnitz University of Technology, Professorship Micromanufacturing Technology, Reichenhainer Str. 70, 09126 Chemnitz, Germany
}

\begin{abstract}
Micro forming can be used for a highly efficient manufacturing of small parts like screws or caps. Because of the small dimensions of the structures, high stresses and forming forces occur, especially in the case of micro bulk forming. In addition, the influence of friction is significantly higher compared to macro forming processes. A typical example of small structures are micro channels. An approach for the reduction of the necessary forming force to obtain lower loads on the tool consists in a vibration-assisted micro forming process. As vibration source, a piezo power module is placed directly in the force axis of the forming press. For the investigations, micro channels with a depth of more than $300 \mu \mathrm{m}$ and a width of $300 \mu \mathrm{m}$ are formed into $1.5 \mathrm{~mm}$ thick AlMg4.5Mn0.7 aluminium alloy sheets. The focus of the research lies in the influence of the process parameters like frequency and oscillation amplitude onto the material flow and the achieved channel depths. To investigate a possible influence on the friction conditions due to the vibration assistance, different lubrication conditions are applied. First results show a channel depth increase of $12 \%$ compared to samples formed without vibration assistance.
\end{abstract}

Keywords: Micro forming, Aluminium, Vibration assistance

\section{Introduction}

Micro forming is a highly efficient and precise manufacturing method for small parts as well as for small functional structures. Such small parts can be screws, bolts, caps, or other structures like frames, mostly used for electronic devices. In addition, micro forming also allows the generation of small structures into the surfaces for a functional integration. Typical examples are micro grooves or channels that can be used for micro fluidic applications, micro cooling systems, or for the direct integration of active materials like piezo ceramics to generate smart sheets with integrated sensor and actuator functionalities.

Because of the small dimensions of the structures, high stresses and forming forces occur, especially in the case of micro bulk forming of micro channels. In addition, the influence of friction between tool and workpiece is significantly higher compared to macro forming processes.

A reduction of the necessary forming force due to a decrease of the friction leads to a more intense material flow, a higher achievable precision, and a reduction of the tool load. One approach for the reduction of the friction forces is a vibration-assisted micro forming process. Researches show, that ultrasonic vibration assistance can lead to a reduction of the forming force [1], as well as a reduction of surface roughness values [2], or nearly an elimination of the spring back behaviour [3]. These researches are mainly focused on the generation of single micro parts or investigations in micro deep drawing. The applied forming force of those processes is lower, compared to bulk forming.

The application example of this research is the generation of an array of ten micro channels, which is intended to serve as intake cavities for piezo rods. Subsequently, the rods can be placed into the channels. After a joining by forming process, the integrated piezo ceramic rods in combination with the aluminium sheet is supposed to act as a smart sheet with sensor and actuator functionalities [4]. Because of forming forces of $80 \mathrm{kN}$ and above, large high-powered ultrasonic vibration systems would be necessary. Due to this, the paper focuses on the investigation, how a low frequency vibration assistance can improve the forming process.

\section{Experimental procedure}

The micro channel array consists of ten single channels with a channel width of $300 \mu \mathrm{m}$, a channel depth of $300 \mu \mathrm{m}$, and a channel length of $10 \mathrm{~mm}$. Within one press stroke also secondary cavities, which are placed perpendicular and in parallel to the micro channels, are generated. The cavities, positioned parallel to the micro channels act as flow barriers to decrease the lateral material flow, whereas the perpendicular to the channels positioned cavities act as space for electrodes of the piezo fibres. The entire area of the micro structure is $7.2 \times 14 \mathrm{~mm}^{2}$. Fig. 1a) shows the micro channels and the secondary cavities. A cross section of the micro channels is represented in Fig. 1b).

\footnotetext{
*Corresponding author: benedikt.mueller@mb.tu-chemnitz.de
} 

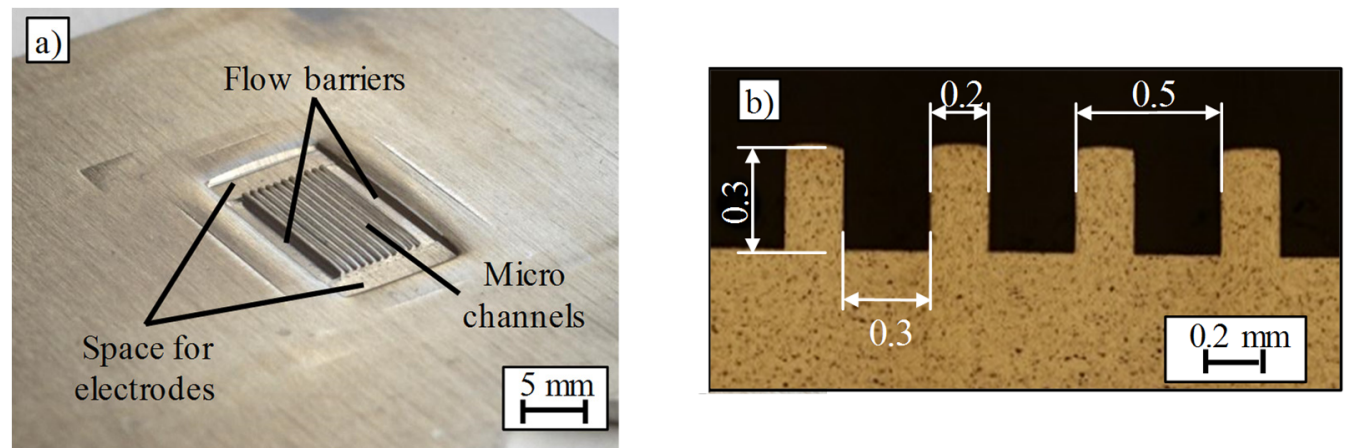

Fig. 1. a) Geometry of the samples; b) Cross section and dimensions of the micro channels.

The micro forming tool, used for this investigation, consists of a upper and a lower die. The tool stamp is made from hardened (64 + 2 HRC) HS6-5-2C (1.3343) high speed steel and is designed as the negative structure of the micro channels. Together with a blank holder, which is set to a blank holder force of $2 \mathrm{kN}$, the stamp forms the upper die. The test samples are $50 \times 50 \mathrm{~mm}^{2}$ aluminium (EN AW-5083 - AlMg4.5Mn0.7) plates cut out of $1.5 \mathrm{~mm}$ thick sheets using water jet cutting. The samples are placed on a flat lower die plate, which is set on top of a piezo power module, acting as vibration source. The power module of the company CeramTec consists of several piezo stack actuators housed in a hermetically sealed steel case. The module is capable of reaching a frequency $f=50 \mathrm{~Hz}$ and can be used up to a blocking force of $F=80 \mathrm{kN}$. Up to this force, the piezo power module is able to deliver an oscillation amplitude. A sine wave is used as input signal for the oscillation.

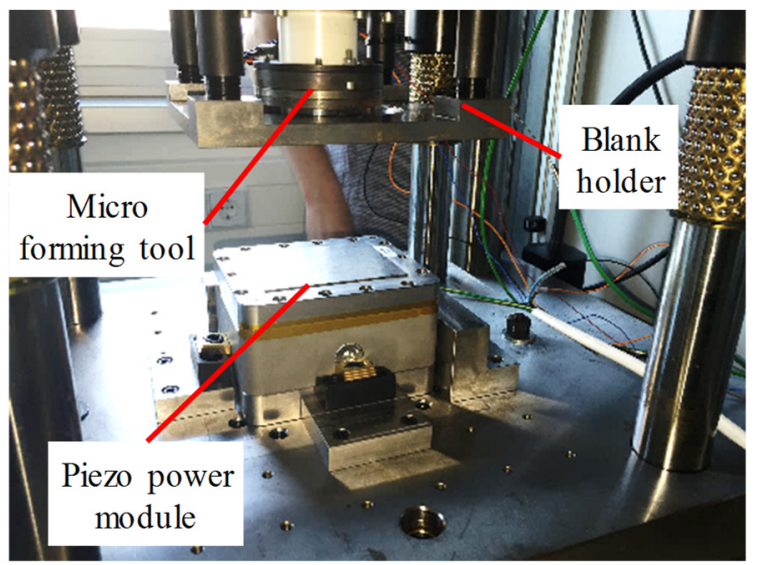

Fig. 2. Micro forming tool and piezo power module (lower die plate not shown).

All the components are integrated into a $200 \mathrm{kN}$ precision servo press P.FÜ.MA from the company Hegewald \& Peschke.

A cleaning step with ethanol is performed on the samples before they are lubricated with oil and placed on the lower die plate. After each forming experiment, the tool and the sample are cleaned again. In a next step, the characterisation of the samples is conducted. Therefore the channel depth $d_{c}$ is chosen as evaluation parameter to obtain information regarding the material flow. For the measurements a Nikon MM400 measuring microscope is used. To visualise the forming results, additional profile scans are made using a 3D laser scanning microscope. The profile data of each measured sample consist of 60 single images, which are stitched together with a special software. To gain comparable results the image processing parameters and scales are held constant for all measurements.

For the experiments, the parameters, shown in Table 1 are varied and their influence on the channel depth and the material flow are investigated. All forming experiments are carried out with a constant stamp velocity $v_{s}$ up to a maximum forming force of $F=70 \mathrm{kN}$. For each parameter set, two samples are formed.

The forming force is below the blocking force of the power module to ensure that a vibration amplitude is generated during the forming process, even if complete tool filling is not achieved at this point.

Table 1. Investigated parameters.

\begin{tabular}{lll}
\hline Parameter & Symbol & Specification \\
\hline Lubrication condition & - & $\begin{array}{l}\text { Plantoform, } \\
\text { Platinol }\end{array}$ \\
Frequency & $f$ & $(10 ; 20 ; 50) \mathrm{Hz}$ \\
$\begin{array}{l}\text { Amplitude of sine input } \\
\text { signal }\end{array}$ & $y$ & $(1.25 ; 2) \mathrm{V}$ \\
\hline
\end{tabular}

For technological reasons, the maximum frequency of the piezo power module is limited to $20 \mathrm{~Hz}$ at an amplitude of $2 \mathrm{~V}$ and above. To prevent a negative input signal, an offset of $5 \mathrm{~V}$ is applied to the input sine signal.

Furthermore, as a reference, samples are formed without vibration assistance. As lubricants, two special forming oils are used, whereas the viscosity is different between both types. With $49 \mathrm{~mm}^{2} / \mathrm{s}$ Plantoform MBO 2797 from the company Fuchs exhibits the lower viscosity compared to the viscosity of $163 \mathrm{~mm}^{2} / \mathrm{s}$ from Platinol V 1937/11 manufactured by OEST.

\section{Results and discussion}

During the forming process up to the forming force $F=70 \mathrm{kN}$ the stresses in the sample are not sufficient to generate a complete uniform channel array field. This can be seen in Fig. 3, indicated with the dashed line. 


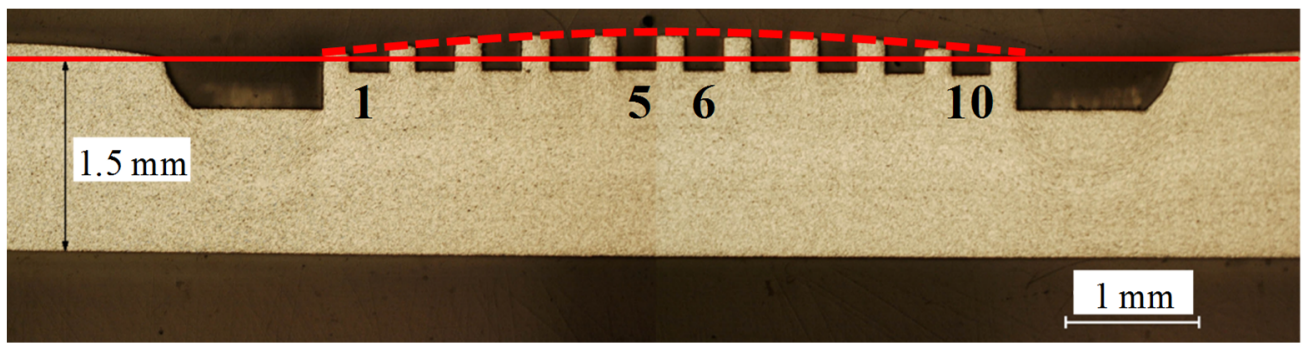

Fig. 3. Section of a formed aluminium sheet with non-homogeneous formed channels.

When the tool is pressed on top of the aluminium sheet, a stress state is generated, which leads to a material flow into the opposite direction of the applied forming force. The solid line in Fig. 3 shows the initial sheet thickness and how the material, forming the webs of the structure, flows into the die. The discussion in this paper will focus on this part of the material flow, which flows into the die to form the channels. All the lateral material flow is not part of this evaluation.

At the beginning of the forming process, the material flow starts in the middle of the structure (channels 5 and 6, see Fig. 3). The highest structure depths are achieved for these channels. Conversely, at the latest, the deformation starts at the edge of the structure (channel 1 and 10), which manifests in the lowest channel depths. In the following evaluation, the maximum channel depth represents the deepest channel of a sample and the minimum channel depth the lowest channels from the edge of the structure. All measurements are conducted at a channel length of $5 \mathrm{~mm}$, which is the lateral centre of the structure.

Fig. 4 and Fig. 5 show the mean minimum channel depths and the mean maximum achieved channel depths for the different vibration parameters, using Plantoform as forming oil. The dashed line in both diagrams stands for the mean minimum or maximum channel depth of the reference samples, formed without vibration assistance. All other parameters like forming force or stamp velocity are the same as for vibration-assisted experiments.

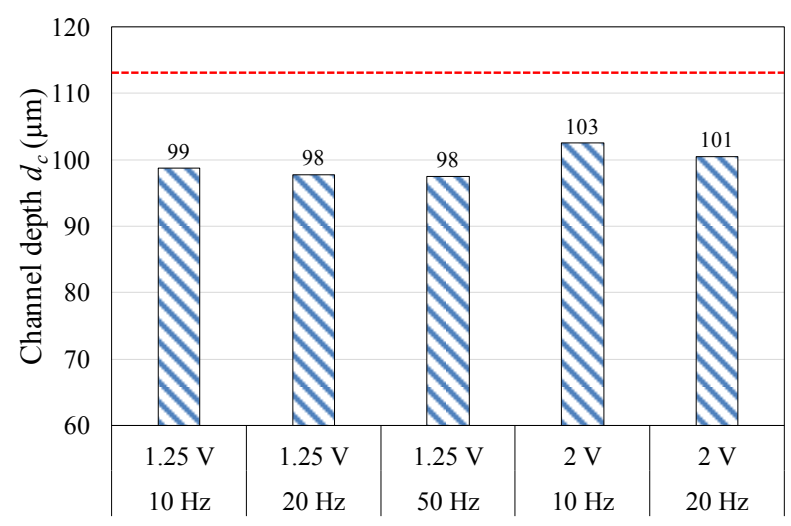

Fig. 4. Minimum channel depth according to amplitude and frequency for samples formed with Plantoform.

The minimum mean channel depth of the reference is $d_{c}=112 \mu \mathrm{m}$ and the maximum mean channel depth is $d_{c}=217 \mu \mathrm{m}$. As can be seen in Fig. 4, the minimum channel depths for samples formed with Plantoform are in a very narrow range from $98 \mu \mathrm{m}(1.25 \mathrm{~V}$ and $50 \mathrm{~Hz})$ to
$103 \mu \mathrm{m}(2 \mathrm{~V}$ and $10 \mathrm{~Hz})$. Because of these nearly identical values, only a slight trend can be observed for this measurement series. By varying the frequency $f$, a slight decrease of the achieved channel depth can be observed for increasing frequencies. This small trend applies both to an amplitude of $1.25 \mathrm{~V}$ and to an amplitude of $2 \mathrm{~V}$. Furthermore, it can be seen that higher minimum channel depths are achieved with an increase of the oscillation amplitude y. However, it is very noticeable that the minimum depths of all samples formed with vibration assistance are significantly below the reference $(9 \%-14 \%)$.

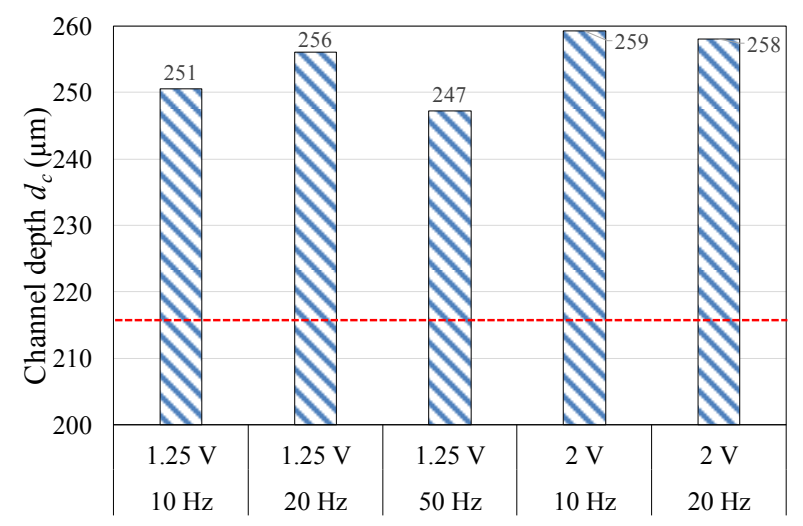

Fig. 5. Maximum channel depths according to amplitude and frequency for samples formed with Plantoform.

When comparing the trends in Fig. 4 with the maximum channel depths in Fig. 5, it is obvious that there is no clear influence of the frequency onto the channel depth. The results of vibration assisted forming experiments using Plantoform show maximum channel depths ranging from $d_{c}=247 \mu \mathrm{m}$ to $d_{c}=259 \mu \mathrm{m}$. Both results achieved with the higher oscillation amplitude indicate slightly higher channels depths, which corresponds to the minimum depths of this test series.

But at the centre of the structure, which is represented by channels 5 and 6 or their depths, all samples formed with vibration assistance samples show a significant increase of the depth compared to the reference samples. An increase in channel depth of about $14 \%-19 \%$ is observable. This nearly correlates to the $9 \%-14 \%$ deeper channels of the reference channels in the border region of the structure. So at this point, it is not clear if the vibration assistance with low frequencies has a positive influence on the forming process. Therefore, Table 2 shows the overall mean channel depth of all channels of an array for each parameter setup. To gain information about the distribution of the achieved channel depths and 
so the uniformity of the structure, the difference $\Delta d_{c}$ from maximum to mean channel depth is calculated. A very small difference between the maximum and the mean channel depth indicates a uniform forming result. In contrast, a large difference indicates an uneven shape of the structure. Furthermore, Table 2 shows the increase in overall mean channel depth compared to the reference.

It can be seen in Table 2 that the overall mean channel depths can be increased about $5 \%-12 \%$ by a vibration assistance.

Table 2. Averaged results for geometry of samples formed with Plantoform.

\begin{tabular}{|c|c|c|c|}
\hline & $\begin{array}{c}\text { Overall mean } \\
\text { channel depth }(\mu \mathrm{m})\end{array}$ & $\Delta d_{c}(\mu \mathrm{m})$ & Increase \\
\hline Ref. & 170 & 47 & - \\
\hline $\begin{array}{c}1.25 \mathrm{~V} \\
10 \mathrm{~Hz}\end{array}$ & 182 & 69 & $7.1 \%$ \\
\hline $\begin{array}{c}1.25 \mathrm{~V} \\
20 \mathrm{~Hz}\end{array}$ & 184 & 72 & $8.2 \%$ \\
\hline $\begin{array}{c}1.25 \mathrm{~V} \\
50 \mathrm{~Hz}\end{array}$ & 179 & 68 & $5.3 \%$ \\
\hline $\begin{array}{c}2 \mathrm{~V} \\
10 \mathrm{~Hz}\end{array}$ & 191 & 68 & $12.4 \%$ \\
\hline $\begin{array}{c}2 \mathrm{~V} \\
20 \mathrm{~Hz}\end{array}$ & 186 & 72 & $9.4 \%$ \\
\hline
\end{tabular}

Beneath the increase of the channel depths through the vibration assistance, also the material flow is changed. The reference samples show shallower channels, but a more even channel depth distribution over the entire array. This fact can be seen from the parameter $\Delta d_{c}$, which is shown in Table 2. The difference between maximum and overall mean channel depth for samples formed with vibration assistance is higher, but in a small range of $4 \mu \mathrm{m}$ $(68 \mu \mathrm{m}-72 \mu \mathrm{m})$.

To illustrate the different material flow, profile scans are made, using a 3D laser scanning microscope. Two scans represented in Fig. 6. On the upper profile, a reference sample, formed without vibration assistance is shown, whereas the lower figure represents a sample formed with an oscillation amplitude of $y=2 \mathrm{~V}$ and a frequency of $f=10 \mathrm{~Hz}$. In the figure, all channels and the adjacent flow barriers are shown and measured. Due to this, the indicated maximum channel heights are nearly $400 \mu \mathrm{m}$ because the height measurement starts in the areas of the deeper flow barriers.

The figures confirm the measurement results that the outer webs of the structure are slightly higher in the reference sample, resulting in deeper channels in this area. Also the benefit of the vibration assistance is clearly visible in the figures. The red and nearly white coloured webs in the lower figure point at significant higher webs and deeper channels than the reference sample. An explanation for this behaviour can be found in the friction. When the material flow starts in the centre region, the material flows into the die and forms the first webs of the structure.
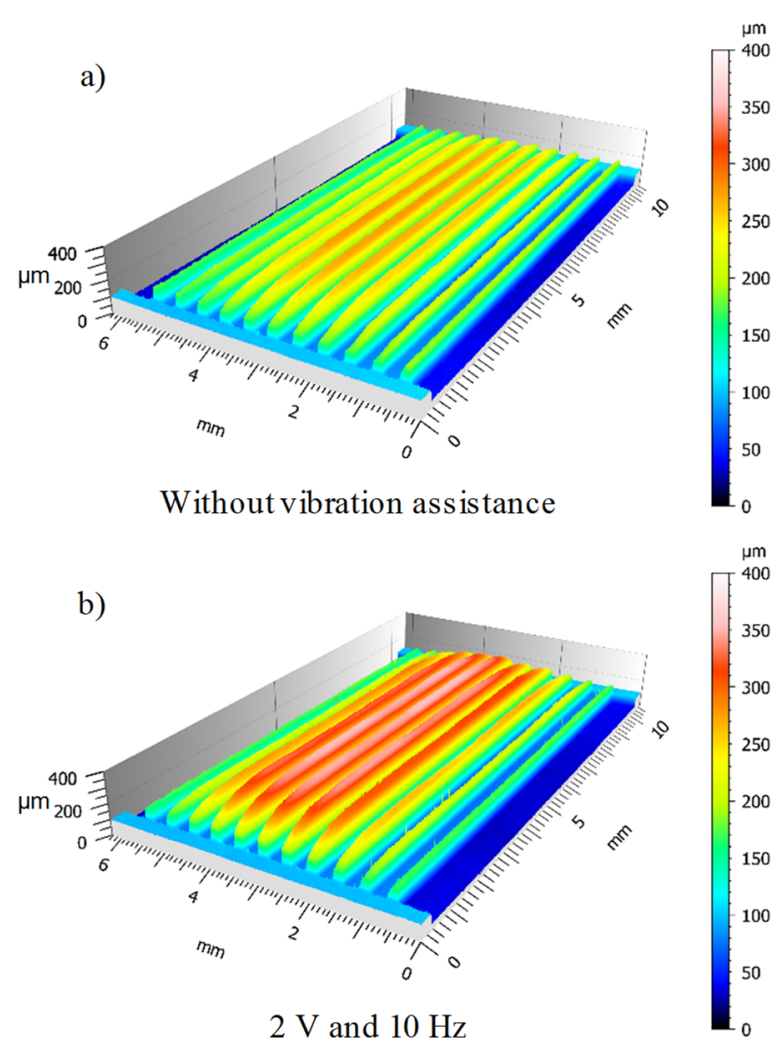

Fig. 6. Profile scan of samples a) Formed without vibration assistance b) Formed with vibration assistance.

The material comes in contact with the sidewalls of the webs in the die, which leads to an increase of friction at this moment. This lowers the material flow, increases the induced stresses and leads to a sideways material flow into other channels in the border region of the structure. With the relative motion during the vibration assisted forming process, the tool and the workpiece are separated again for a little moment, which has a favourable effect on the friction conditions. So the material flow into the centre channels of the die is larger compared to the reference sample and the forming of the outer webs starts later. Due to the fact, that every experiment is done until the maximum force of $F=70 \mathrm{kN}$, in both cases the generated stresses are not high enough to lead to a material flow, which fills the die completely.

The same experiments are also done with Platinol, a forming lubricant with a higher viscosity. It could be possible, that during the relative motion of the tool, more lubricant is able to flow into the contact area to support the forming process. It can be assumed, that with "thicker" oil, this phenomena would not happen in such a significant way. The results regarding the minimum and maximum channel depths of samples formed with Platinol are shown in Fig. 7 and Fig. 8. 


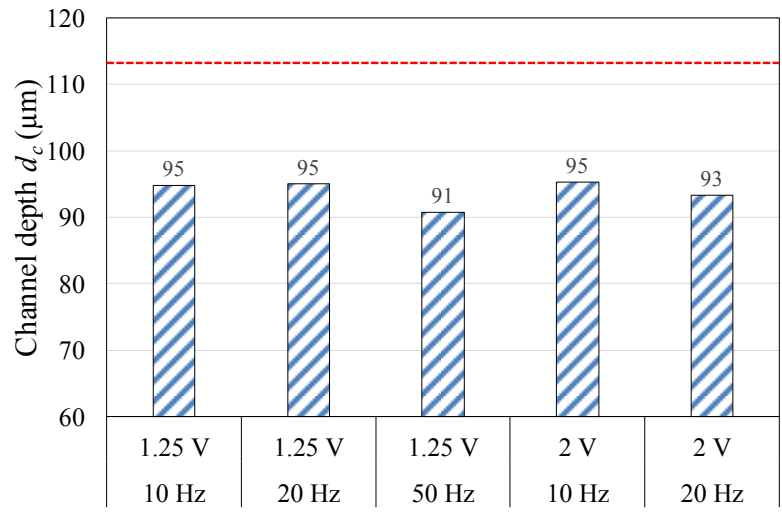

Fig. 7. Minimum channel depth according to amplitude and frequency for samples formed with Platinol.

Similar to the results with Plantoform, the minimum channel depths are also below the reference for the samples formed with Platinol. For no parameter combination, channel depth exceeds $d_{c}=95 \mu \mathrm{m}$, whereas this is the case for all experiments with Plantoform. The same behaviour can also be seen in Fig. 8. Even the highest channel depth of the Platinol test series $\left(d_{c}=247 \mu \mathrm{m}\right)$ is not larger than the lowest value achieved with Plantoform. Nevertheless, all maximum channel depths of samples formed with vibration assistance using Platinol are above the reference value.

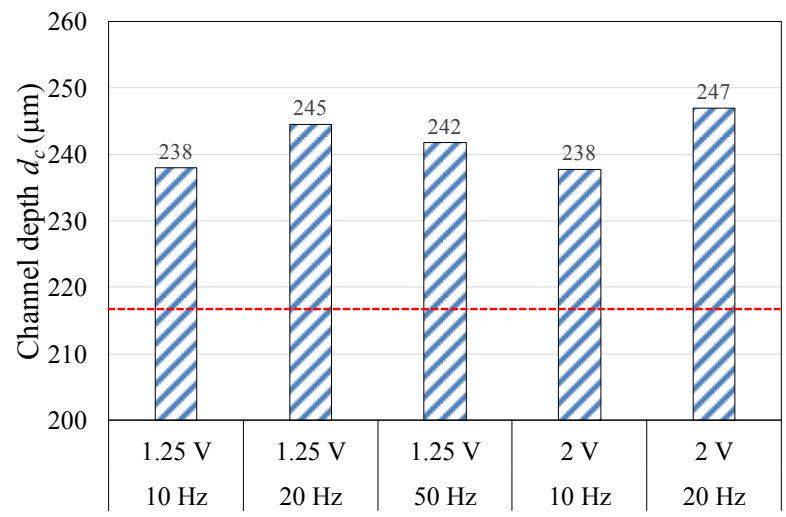

Fig. 8. Maximum channel depth according to amplitude and frequency for samples formed with Platinol.

The overall mean channel depths of all ten channels, shown in Table 3, indicate that the thicker oil (Platinol) lead to almost no improvement due to vibration assistance.

Table 3. Averaged results for geometry of samples formed with Platinol.

\begin{tabular}{|c|c|c|c|}
\hline & $\begin{array}{c}\text { Overall mean } \\
\text { channel depth }(\mu \mathrm{m})\end{array}$ & $\Delta d_{c}(\mu \mathrm{m})$ & Increase \\
\hline Ref. & 170 & 47 & - \\
\hline $\begin{array}{c}1.25 \mathrm{~V} \\
10 \mathrm{~Hz}\end{array}$ & 175 & 63 & $2.9 \%$ \\
\hline $\begin{array}{c}1.25 \mathrm{~V} \\
20 \mathrm{~Hz}\end{array}$ & 180 & 65 & $5.9 \%$ \\
\hline $\begin{array}{c}1.25 \mathrm{~V} \\
50 \mathrm{~Hz}\end{array}$ & 172 & 69 & $1.2 \%$ \\
\hline $\begin{array}{c}2 \mathrm{~V} \\
10 \mathrm{~Hz}\end{array}$ & 174 & 63 & $2.4 \%$ \\
\hline $\begin{array}{c}2 \mathrm{~V} \\
20 \mathrm{~Hz}\end{array}$ & 178 & 69 & $4.7 \%$ \\
\hline
\end{tabular}

By investigating the profile scans of samples formed with Platinol (Fig. 9), it can be seen, that the material flow in the lateral direction is not as homogenous compared to the reference or Plantoform samples (Fig. 6). At the channel length of about $10 \mathrm{~mm}$, the achieved channel depths are higher, compared to the centre of the structure.

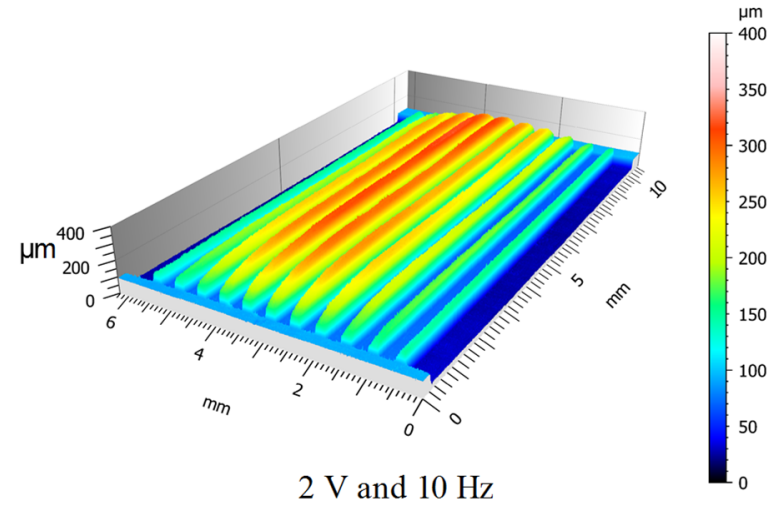

Fig. 9. Profile scan of a sample formed with vibration assistance and Platinol.

\section{Summary and conclusions}

Experiments concerning vibration assisted micro forming of channels into aluminium sheets are conducted. Therefore, lubrication conditions, the oscillating amplitude and the frequency are varied and their influence on the achievable channel depth and the material flow is studied.

The vibration assistance in combination with the lowviscosity forming lubricant Plantoform shows a significant improvement regarding channel depth compared to reference samples. A slight trend that a higher amplitude can lead to deeper channels was found. Because of the unfinished tool filling, higher deviations $\Delta d_{c}$ occur. Due to this, more experiments should be done to improve the statistically reliability of the data.

In summary, it can be stated that with the vibration assistance a larger deformation or material flow can be achieved. Measured by the geometric parameter channel depth $d_{c}$, the improvement is slightly more than $12 \%$.

This research was supported by the Deutsche Forschungsgemeinschaft (DFG) in context of the Collaborative Research Centre/Transregio 39 PT-PIESA, subproject A2.

\section{References}

1. Y. M. Huang, Y. S. Wu, J. Y. Huang, Int. J. Adv. Manuf. Technol. 71, 1455-1461 (2014)

2. Y. Bai, M. Yang, Proc. Eng. 81, 1475-1480 (2014)

3. S. Amini, A. H. Gollo, H. Paktinat, Int. J. Adv. Manuf. Technol. 90, 1569-1578 (2017)

4. B. Müller, A. Schubert, Proc. Eng. 207, 1045-1050 (2017) 